\title{
Editorial
}

\section{Robotics in Medicine}

Robots have entered mainstream surgery. There are quite a few misconceptions about robots and their applications. To begin with the term, robot is in itself a misnomer for the current set of robots available as they are not truly autonomous. They are supercomputers with mechanical control over very tiny instruments. The current 'robot' represents a slavemaster relationship.

What are the advantages of robots or 'robotics' in current surgical situations?

They are found to be particularly useful in tight corners of the body, like the prostate, thorax and areas, where there is very little room for maneuvering.

$3 \mathrm{D}$ vision is a huge advantage. Conventional laparascopic surgery is two-dimensional. The da Vinci system has two lenses separated in such a way so as to give a different perspective of the same field thus creating a 3D picture of the same field. This gives the surgeon the impression of actually being there in the surgical field.

Visual magnification is also vastly improved and offers a visual magnification of 10 to 15 times greater than normal. The images are also much brighter as the telescope is bigger $(12 \mathrm{~mm})$.

Motion scaling is another major advantage. A small motion outside the body can cause an amplification of motion at the instrument tip. The da Vinci robot filters and deamplifies this up to a scale of 5 to 1 . This eliminates tremors of the hand. So, a surgeon with tremors working at the console will have his tremors removed by the robot. Large movements are converted to micromotions. Large motions of control grips are reduced to micromotions.

In conventional laparascopy, the surgeon will need to stand and may experience discomfort if he has to stand for long periods of time. For robotic surgery, the surgeon can sit in a comfortable position at the console.

Endowrist technology allows the daVinci system capability of spinning a full two revolutions against the human wrist of $270^{\circ}$. The robot can rotate and angulate in several different directions and thus offers unparalleled dexterity. The robot offers precision. This allows for the correct identification of vital structures which can be clearly seen and either targeted or avoided. Robotic surgery offers smaller incisions, less scarring, decreased blood loss, shorter hospital stays and a faster return to active life. Fewer hospital staff are required when a robotic surgery is being conducted.

Telesurgery is thought to be another potential benefit. Thus, a surgeon can operate on a patient who may be situated in a different location from the surgeon. At this time though, in telesurgery, there is a lag or latent period in response to the surgeon's movements from the console. So, for the current time, the surgeon has to be located at the console controlling the robot inside the operating room. But, this offers future possibilities especially once robots improve, evolve and get more sophisticated.

The da Vinci robot currently available costs 1 million US dollars and its maintenance is equally expensive. Are robots likely to become less expensive or more expensive in the future? Some experts say they will become less expensive. Others, quote haptics, increased processor speeds, more complex and capable software as factors that will cause robots to become more expensive. The other concern is that once a system is purchased can it be upgraded with minimal costs? The robots at this time leave large footprints and are relatively cumbersome. Operating rooms have to be redesigned to accommodate them. Many operations need to be reconfigured if robotic surgery is to be performed.

What is the downside to robotic surgery?

Many surgeons and critics agree that, in the right hand, the da Vinci robot is generally safe tool (robot) with which to perform surgery.

That being said it has been found that robotic surgery takes longer and costs much more. Many critics complain that industry often speaks on behalf of the hospitals and make unsubstantiated claims. Often, there are serious conflict of interest questions. At times, there are claims of misinformation. In an article, in JAMA, it was found that to date robotically-assisted hysterectomy has not been found to be more effective than laparascopic surgery. ACOG (American College of Gynecologists) President James T Breeden issued a statement on March 14, 2013, on robotic surgery and is worth reading in full. He states that expensive technology for routine surgery does not improve patient outcomes. He further states 'vaginal and laparascopic hysterectomy shortens hospital stays, less discomfort, faster return to function and have proven track records for improving patient outcomes and cost efficiency'. He, however, acknowledges that there is scope for robotic surgery in unusual and complex clinical conditions. 
Sensory input from the robot is nonexistent and the surgeon does not get the feel of tissues.

Problems associated with robotic surgery are burns and heat-related damage to the intestines and ureters. Many critics think that injuries and complications related to robotic surgery have not been reported to the FDA resulting in an under reporting of adverse events related to robotic surgery. In other words, complications related to robotic surgery are far more than reported.

Despite this data is growing. Currently, robotic surgery is most useful in intra-abdominal microsurgery. Manipulation in very small spaces in outstanding, especially in the field of cardiac surgery and sutureless anastomoses. Pediatric laparascopic surgery is another avenue that is opening up to robotic surgery.

\section{The Future}

Robotic systems are information systems and thus have the ability to interface and integrate with many emerging and evolving technologies. Fusing preoperative images (through CT scans and or MRI) with intraoperative image videos to guide the surgeon to better dissection and improved identification of pathology are future goals. At the same time, instruments need to be developed to incorporate the full range of sensory input. Other areas of expansion for the robot are diagnostic testing and evaluation, ultrasonography, ear infrared and confocal microscopy.

I personally feel that robots are here to stay. Over time, a new generation of robots will evolve as will a new breed of surgeons who will be completely familiar with operations performed through the use of a robot. As I stated right in the beginning, the robots of today function on a slave-master relationship. Time will decide who is the master in this relationship.

Chris de Souza

MS, DORL, DNB, FACS

Honorary ENT and Skull Base Surgeon

Tata Memorial Hospital

Consultant, ENT-Head and Neck Surgeon

Lilavati Hospital and Holy Family Hospital, Mumbai, Maharashtra, India 